\title{
Sprachliche Entfremdung als Phänomen des Umbruchs in der früheren DDR
}

\author{
Hans Ester \\ Vakgroep Algemene Kunstwetenschappen \\ Katholieke Universiteit \\ NIJMEGEN
}

\begin{abstract}
Linguistic alienation as phenomenon of the transformation in the erstwhile DDR

The Federal Republic of Germany and the German Democratic Republic have not yet grown into a coherent unity since the political unification of the two 'Germanies' in 1990. The reason for the lack of sympathy and the irritations on both sides possibly lies in the fact that the actual developments did not meet the general expectations during the first years after 1990. The thesis of my article is that the more profound reasons for the alienation between the western and the eastern part of Germany can be found in the little interest on the western side for the developments in the GDR from 1949 until the fall of the Wall in 1989. The lack of interest in the forty years of the GDR's existence finds its expression in the alienation of language. In order to improve communication between the Germans of both spheres, the reading of literary texts from the former GDR by members of the entire new Federal Republic of Germany can be a reconcilliatory device. In this way the reader can obtain insight into forty years of history.
\end{abstract}

Die Athletin Katrin Krabbe aus Neubrandenburg wurde wegen Dopingverdachts Opfer der Sensationshascherei der westdeutschen Presse. Katrin Krabbe ist aber im Gegensatz zu vielen ihrer früheren DDR-Landsleute nicht auf den Mund gefallen. In einem Interview mit der Beilage Zeit-Magazin wehrte sie sich tapfer gegen die Angriffe und bezog auch die DDR, das Land ihrer Geburt, in ihre Reaktionen ein:

Es gibt Dinge, die auch in der DDR gut waren. Da stehe ich voll zu. Mir ist vorgeworfen worden, da $\beta$ ich bei der Siegerehrung mit der bundesdeutschen Hymne nichts anfangen konnte. Aber ich bin nun mal mit der DDR-Hymne großgeworden, ich kannte keine andere Hymne. Auf einmal stehst du da oben und es läuft eine fremde Hymne (Krabbe, 1993).

Man wei $\beta$ nicht, wen man mehr zu bedauern hat: Hoffmann von Fallersleben oder Johannes R. Becher, die alte oder die neue Hymne. Es kommt mir aber im 
Grunde gar nicht auf eine Parteinahme oder eine moralische Entscheidung, geschweige denn ein politisches Urteil an. Das Wesentliche an dem von Katrin Krabbe in diesem Interview Ausgesagten ist, da $\beta$ sie (als sie noch die umjubelte Sprint-Weltmeisterin war) in kurzer Zeit eine vertraute Wirklichkeit verlor, und da $\beta$ die Vertreter der nunmehr dominanten neuen Wirklichkeit fraglos davon ausgingen, sie könne sich diesem Neuen problemlos anpassen. Was im Leben der Athletin geschah, die Entfremdung von der vertrauten Wirklichkeit, ist während der letzten sechs Jahre auf den unterschiedlichsten gesellschaftlichen Niveaus unzählige Male geschehen: die DDR war vorbei, nun galt es die böse Erinnerung an diese leidige DDR zu tilgen. Zum Sprachrohr vieler DDR-Bürger im Kampf gegen den nach der Vereinigung einsetzenden Gedächtnisschwund machte sich die Journalistin Jutta Voigt in einer Reihe von Artikeln in ZeitMagazin. Der Beitrag in der Ausgabe vom 25.10.1991 trägt den unmißverständlichen Titel "Blitzkrieg gegen die Erinnerung".

\section{Verneinung der Vergangenheit führt zur Entfremdung}

Wenn im Leben eines Menschen etwas negiert wird, was eindeutig negative Aspekte hatte (oder sogar in seiner Substanz negativ war), dann kann die Reaktion des seiner Vergangenheit beraubten Menschen darin bestehen, da $\beta$ er diese Vergāngenheit als das ihm Eigene zu verteidigen beginnt. Diese Möglichkeit liegt nahe, wo es um die einstige Deutsche Demokratische Republik geht. Krabbes Klage über das mangelnde Verständnis auf westdeutscher Seite für die Identitätsprobleme der ehemals sich als Bürger der DDR verstehenden Menschen bildet eine allgemeine soziale Erscheinung. Sie ist nicht auf damals gefeierte und privilegierte Sportler und Künstler beschränkt. Deswegen soll diese Klage ernstgenommen und als Symptom einer allgemeinen Identitätskrise verstanden werden. Wie breit gefächert dieser Unmut ist, läßt sich zum Beispiel an den Leserbriefen in der Tageszeitung Neues Deutschland ablesen. Einsicht in die Ursachen dieser Identitätskrise ist für die Zukunft de: Bundesrepublik Deutschland eine lebensnotwendige Angelegenheit. Der frühere Bundeskanzler Helmut Schmidt hat das komplexe Problem im Jahre 1993 hervorragend zusammengefa $\beta$ :

Vor allem haben viele Menschen der ehemaligen DDR etwas in das vereinte Deutschland mitgebracht, was niemand im Westen besitzt, nämlich eine besondere Erfahrung im Umgang mit sich selbst und die Erinnerung an den solidarischen Zusammenhalt in Zeiten seelischer und materieller Not und Entbehrung. Die Westdeutschen müssen deshalb unterscheiden zwischen dem gescheiterten politischen und okonomischen System der DDR einerseits und dem in der DDR gelebten Leben ihrer Landsleute andererseits. Diese Leben sind nicht gescheitert (Schmidt, 1993). 
Die Wahrheit lautet, da $\beta$ es bisher wenigen vergönnt war, diese Unterscheidung zu machen.

$\mathrm{Da} \beta$ man die politischen Entwicklungen vollauf begrüßen kann, die zum Ende der Herrschaft der Sozialistischen Einheitspartei Deutschland geführt haben und dennoch über den Verlust des Wertvollen in der DDR trauern kann, beweist der Wittenberger Pfarrer Friedrich Schorlemmer mit seinem Buch Worte offnen Fäuste (1992). Da Schorlemmer (geb. 1944) von seiner Schulzeit an ein Opponent der SED-Politik gewesen ist, hat sein Wort über die DDR einen Wert, der die Färbung des Parteilichen weit übersteigt. Es ist notwendig, da $\beta$ wir dieses Wort hören, das auf Versöhnung und nicht auf Konfrontation zielt.

Über wenig andere Bürger der DDR hat die Staatssicherheit so genau und ausführlich Buch geführt wie über Friedrich Schorlemmer. Als er Einblick in seine Stasi-Akte bekam, zeigte sich, daß der Pfarrer der Schloßkirche in Wittenberg von scheinbar loyalen Mitarbeitern, in Wahrheit aber von einem Heer von Informanten umgeben gewesen war. Was hätte mehr nahegelegen als eine totale Distanz zur Vergangenheit dieses DDR-Staates? Das war aber nicht Schorlemmers Weg. Im ersten Teil seines Buches mit dem Titel "Die Rückkehr in ein schwieriges Vaterland" legt Schorlemmer anhand von offiziellen Briefen, von Berichten über Gespräche mit Vertretern des Staates und der Kirche und mit Hilfe von Predigten Verantwortung ab über seine Haltung während zweier Jahrzehnte DDR. Diese Verantwortung macht deutlich, was fur ihn und viele andere evangelische Christen in der DDR das Hauptproblem ihres Lebens war: Wie mache ich unter den gegebenen Umständen das Beste aus den mir zur Verfügung stehenden Möglichkeiten, ohne meiner tiefsten christlichen Überzeugung untreu zu werden? Im nachhinein ist Schorlemmer der Meinung, da $\beta$ er zu umsichtig und zu behutsam zu Werke gegangen sei.

Aus dem ersten Teil von Schorlemmers Buch geht unter anderem hervor, da $\beta$ die Geschichte der DDR und infolgedessen die Geschichte Deutschlands anders hätten verlaufen können, wenn die SED in einem frühen Stadium der historischen Entwicklung dem Bedürfnis der DDR-Bevölkerung nach mehr Freiheit entgegengekommen wäre. Nach der Wende im November 1989 fehlte in der DDR die intellektuelle Schicht, die das Vakuum nach dem Wegfall der SED hätte auffuillen können. Oppositionelle Intellektuelle waren ja in der Mehrheit in die Bundesrepublik Deutschland emigriert. Im Fehlen einer DDR-internen intellektuellen Elite sieht Schorlemmer den Grund für das Scheitern einer in der DDR selbst gewurzelten Alternative zur beendeten Herrschaft der DDR. Meine mit Schriftstellern der DDR (Jürgen Rennert, Thomas Brasch, Sarah Kirsch, Kurt Bartsch, Sigmar Schollak) geführten Gespräche haben diese Ansicht bestätigt. Diese Gespräche wurden gesammelt veroffentlicht in Labroisse und Wallace (1991). 
Der zweite Teil von Worte offnen Fäuste umfaßt die Periode November 1989 bis zur faktischen Aufhebung der DDR durch die Währungsunion am 1. Juli 1990. Der dritte Teil des Buches ist der kürzeste der drei und beschäftigt sich mit den Problemen, die im Sog der deutschen Vereinigung entstanden sind:

Der Mielke-Honecker-DDR weine ich keine Träne nach, aber ich trauere dem Aufbruch in eine Deutsche Demokratische Republik nach, dem aufkommenden Selbstbewußtsein, dem Selbstprüfungs- und Selbstläuterungsproze $\beta$ nach schwierigen vierzig Jahren mit Hoffnung und Scheitem, mit Kleinkariertem und Großspurigem, mit großem und kleinem Theater, mit tragenden Zeilen und aufrüttelnden Bildern, mit dem großen Dialog des Volkes, der plötzlich ohne die öden Rituale der Vergangenheit begonnen hatte. Der euphorische Flug in die Einheit wurde zum Sturzflug, nicht nur für die Wirtschaft. Ihm fehlte der ihn beflügelnde Geist. Die Ungeduld war zu gro $\beta$. Einerseits war die ökonomische Basis schon zu zerstört, und andererseits waren die konsumorientierten Sehnsüchte der Bürger schon zu gro $\beta$. Aber geben Bockwurst und Banane schon ein Ganzes, wenn sie wie zwei Hälften zusammengefügt werden? Der juristischen, völkerrechtlichen, politischen und wirtschaftlichen Vereinigung und Gleichstellung mu $\beta$ erst noch die menschliche folgen. Die gegenseitige Verständigungsaufgabe liegt vor uns. Wir haben den sichtbaren Graben übersprungen und sind im Unsichtbaren angekommen. Wir werden noch viel Offenheit und Geduld brauchen. Wir werden nicht alles verstehen können, weil nur wirklich verstehen kann, wer das Schicksal geteilt hat (Schorlemmer, 1992:379).

Im Hinblick auf die Theorie und die Praxis des Marxismus kann man Friedrich Schorlemmer kein $X$ für ein $U$ vormachen. Für ihn ist der Stalinismus systemimmanent und auch bereits vorgegeben in den Werken von Karl Marx. Die Liquidierung der DDR führt nun aber zum Verschrotten dessen, was der Mühe einer Sichtung und Überprüfung wert gewesen wäre. Worin lag für Schorlemmer das Wertvolle? Er spricht vom authentischen Verlangen nach Wahrheit und Solidarität, das in der DDR neben allen anderen Dingen vorhanden war:

Der Wert der Wahrheit war uns teuer. Manchen kam sie teuer zu stehen. Es ist schon ein Paradox: die Zensur gab dem Wort Gewicht. Und was die Zensur verbot, bekam bei Lesern Gewicht. So diente die Zensur der Wahrheit. So war sie eine List der Wahrheit, an die Menschen heranzukommen. Wo nun alles plötzlich so frei ist, wird alles gleich gültig. Gab es früher Informationshunger, so wird der fast Verhungerte nun überschüttet. Und er überfrißt sich (Schorlemmer, 1992:387).

Am 10. Oktober 1993 hielt Schorlemmer in der Frankfurter Paulskirche eine Rede aus Anla $\beta$ der Verleihung des Friedenspreises des Deutschen Buchhandels 
an ihn. In dieser Rede setzt sich Schorlemmer erneut gegen die "gesamtdeutsche Vergeßlichkeit" zur Wehr (Schorlemmer, 1993:48).

Wenn das Erbe von vierzig Jahren DDR im Handumdrehen für null und nichtig erklärt wird, so ist zwangsläufig die Folge, da $\beta$ die mißbrauchten und irregeleiteten Menschen gegenüber jeglichem Ideal skeptisch werden und in eine harte Amoralität verfallen. Genau dies beobachtet Schorlemmer in der heutigen Zeit. Schorlemmer versucht, zwischen der Scylla der Dämonisierung der DDR und der Charybdis der DDR-Nostalgie hindurchzulavieren. Mag auch sein Stil die Gewohnheit des Predigens durchschimmern lassen, sein Standpunkt ist lauter und wichtig für unsere Zeit. Verständlich wird sein (schon bald nach 1989 überholtes) Plädoyer für eine sanfte Zweistaatlichkeit von West und Ost.

\section{Sprachliche Divergenz zwischen Ost und West}

Die Haltung gegenüber der DDR in der Bundesrepublik Deutschland war anfangs feindselig und ablehnend, später pragmatisch (mit dem Hintergedanken der Erosion der trennenden Mauer) und ist in unseren Tagen (also acht Jahre nach 1989) durch Negation bestimmt. Nie gab es ein wirkliches Interesse der breiten Öffentlichkeit Westdeutschlands für den Bruderstaat im Osten. Dem über die Medien vermittelten Wissen in der DDR über die Gesellschaft der Bundesrepublik entsprach nichts Vergleichbares im Westen. Es existierte zwar ein Buch mit dem Titel Die SBZ von $A$ bis $Z$ (später: $D D R-H a n d b u c h$ )1), aber von fundierten Kenntnissen hinsichtlich der DDR war allgemein gesprochen überhaupt nicht die Rede. Darin liegt der Grund für die auf den ersten Blick erstaunliche Gemütsruhe, mit der in Westdeutschland der politische Übergang der letzten sechs Jahre - für die DDR eine revolutionäre Umstülpung aller Lebensbereiche - aufgenommen wird. Wenn davon ausgegangen wird, da $\beta$ die Menschen in der DDR nichts zu verlieren hatten, so fehlt zwangsläufig die Sensibilität für das Gefühl des Verlustes, der Klage und der Trauer, das viele in der DDR befallen hat.

Auf einer Tagung der Deutschen Akademie für Sprache und Dichtung, die nicht in Darmstadt sondern in Weimar gehalten wurde, stand das Thema "Erschwindelte Bedeutung - ein Gedankenaustausch über unsere Sprache" auf dem Programm. Die Tagung fand im Mai 1991 statt. Nur drei Redner kamen aus dem Osten, unter ihnen der oben vorgestellte Pfarrer Friedrich Schorlemmer.

1 Der erstgenannte Titel machte nach der Gründung der DDR im Jahre 1949 dem kürzeren Titel $A$ bis Z. Ein Taschen- und Nachschlagebuch uber den anderen Teil Deutschlands. 
Auch die Schriftstellerin Angela Krauß hielt einen Vortrag, in dem sie unter anderem folgendes sagte:

Nach der Freude, da $\beta$ wir uns ohne Dolmetscher verstehen, tauchen nun ganz andere Schwierigkeiten auf. Wir machen die Erfahrung, da $\beta$ wir in einer fremden Sprache reden, da $\beta$ manche Wörter für uns anderes bedeuten als für Freunde aus dem Westen (Michaelis, 1991).

An Wörtern nennt Angela Krauß: Friede, Sicherheit, Solidarität. Sie hätte auch andere Wörter nennen können, an denen sich die Geister aus Ost und aus West scheiden mußten, Wörter sowjetischen Ursprungs etwa oder Abkürzungswörter, die auf den Unkundigen den irritierenden Effekt einer Geheimsprache haben. Und auch umgekehrt müßte man Beispiele sprachlicher Art finden, an denen die geschlossene Tür für die Sprecher aus dem Osten sichtbar würde. ${ }^{2}$

Am 26. August 1991 fand in Leipzig ein besonderes Fest statt. An diesem Tag wurde nämlich das "wiedervereinigte", bekanntlich nach Konrad Duden benannte Wörterbuch zur deutschen Rechtschreibung vorgestellt. Der Leiter der Dudenredaktion, Günther Drosdowski, hielt bei diesem Anlaß einen Vortrag, in dem er zur Frage nach dem Ausma $\beta$ der Divergenz der deutschen Sprache im Westen und der deutschen Sprache im Osten bemerkte:

Die mühsam errungene, von Konrad Duden durchgesetzte Einheitsschreibung zerbröckelte nicht in den Jahren, in denen der Duden in eine Leipziger und eine Mannheimer Ausgabe geteilt war, auch das einigende Band der deutschen Sprache zerri $\beta$ nicht in den Jahren der Spaltung, was auch immer über die sprachlichen Folgen der politischen Teilung Deutschlands gesagt oder geschrieben worden ist. Die Besonderheiten, die sich im Laufe der Jahre in der Bundesrepublik und in der DDR herausbildeten, hatten kein großes Gewicht, sie paßten gut unter das gemeinsame Dach, das unsere Hochsprache über den vielfältigen Erscheinungsformen des Deutschen - den Mundarten, den lokalen Umgangssprachen, den Fachund Sondersprachen - bildet. Weder in der Schreibung noch in der Lautung gab es greifbare Unterschiede, auch nicht im grammatischen Bau unserer Sprache, lediglich im Wortschatz, und hier bestanden diese Unterschiede zum Teil nur in den Köpfen der Machthaber und Parteiideologen, die durch die Manipulation der Sprache den neuen sozialistischen Menschen schaffen wollten (Drosdowski, 1991:12).

2 Sehr aufschlußreich sind die Beitrăge, die der Ostberliner Biomathematikcr Jens Georg Reich in der Reihe "Deutsche Ansichten" für Zeit-Magazin schrieb. Ich greife einen Artikel heraus: "Allein, es fehlt die Botschaft" (Reich, 1992). 
Das von Drosdowski Vorgetragene ist denn vielleicht doch ein wenig zu optimistisch gesehen. So leicht låßt sich die Sprache in den Köpfen der Machthaber wohl nicht trennen von der alltäglichen sprachlichen Kommunikation in der DDR. Gewi $\beta$ gab es einen Unterschied zwischen der offiziellen, parteilich normierten Sprache, zum Beispiel des Organs Neues Deutschland, und der im privaten, häuslichen Kreise gesprochenen Sprache. Aber alle Bürger der DDR kamen in Kontakt mit gesellschaftlichen Institutionen, die mit der SED in irgendeinem Zusammenhang standen, so da $\beta$ eine hermetische Abgrenzung gar nicht zu den Möglichkeiten gehörte. Horst Dieter Schlosser kommt in seinem Buch, Die deutsche Sprache in der DDR zwischen Stalinismus und Demokratie historische, politische und kommunikative Bedingungen (1990), zu einer bedeutsamen Schlußfolgenung:

Da sich also die Sprecher in der DDR auch bei Reverenzen vor den offiziellen Sprachnormen stets noch im Rahmen der einen deutschen Sprache bewegten, hat es trotz des Bewußtseins, dabei eine dem Alltag weitgehend entfremdete Sprache zu benutzen, notwendigerweise auch fließende Übergänge gegeben, durch die die Alltagssprache in der DDR sehr wohl beeinflußt wurde. Das ist freilich nicht mehr allein an einzelnen lexikalischen Elementen nachweisbar, die zugleich im offiziellen wie im inoffiziellen Gebrauch galten. Dafür jedoch zwei Beispiele: Allein die Begrifflichkeit der Verwaltung, mit der jeder Bürger in jedem Land notwendige Kontakte hat, gilt nie nur im offiziellen Bereich, sondern auch in der Alltagsrede. Wer in der DDR etwa eine Wohnung suchte, sprach nicht nur offiziell, sondern auch privat von der Kommunalen Wohnungsverwaltung (KWV), deren Dienste er in Anspruch nehmen mußte. Oder: Wer in der DDR Abitur machen wollte, kam auch privat nicht um den Gebrauch des Terminus Erweiterte Oberschule (EOS) herum (Schlosser, 1990:11).

Auf einer anderen Ebene befindet sich der Name Mitropa für die Speisewagengesellschaft der Deutschen Reichsbahn in der DDR. Als eine der sehr, sehr wenigen Ausnahmen hat sich der Name Mitropa auf Kosten der westdeutschen Gesellschaft durchgesetzt.

\section{Der Wortschatz des Ostens}

Wenn es Änderungen sprachlicher Natur zwischen Westdeutschland und Ostdeutschland gegeben hat, so bezogen sie sich weder auf die grammatikalische Struktur noch auf die Syntax. Die Änderungen lagen im Bereich des Wortschatzes. Und diese Änderungen waren nicht auf die deutsche Sprache in der DDR beschränkt. Wenn wir die Situation im Jahre 1945 zum Ausgangspunkt nehmen, müssen wir zunăchst feststellen, da $\beta$ es in bezug auf die Sprache keine Stunde Null gegeben hat. Auch in anderen Bereichen nicht, aber das ist ein 
Thema für sich. In diesem sprachlichen Zusammenhang ist von Bedeutung da $\beta$ der Nationalsozialismus gewisse Kennzeichen des sozialistischen Idioms übernommen hatte. Die Wortverbindungen mit "Volk" bilden ein anschauliches Beispiel: "Volksempfänger", "Volkswagen". Da der Sozialismus marxistischer Prägung Wortbildungen mit "Volk" als wesentlichen Ausdruck seiner Ideologic betrachtete, wollte er nicht auf sie verzichten und schuf damit nach dem Zweiten Weltkrieg eine assoziative Verbindung der kommunistischen Sprache mit dem nationalsozialistischen Idiom. Diese Assoziationen verschafften dem westdeutschen Bürger scheinbar die Legitimierung, sich nicht weiter mit dieser deutschen Volksrepublik, der DDR, zu befassen. Ein anderer Aspekt, den wir in unsere Überlegungen einbeziehen müssen, ist das Ausma $\beta$ der sprachlichen, vornehmlich idiomatischen Änderungen innerhalb der im Westen Deutschland gesprochenen Sprache unter dem Einflu $\beta$ der anglo-amerikanischen Kultur. Es gab ab 1945 also eine Bewegung weg vom Zentrum der gemeinsamen Sprache, an der beide Seiten beteiligt waren. Diese Bewegung beschränkte sich auf den Wortschatz und berührte dabei nur einen relativ geringen Prozentsatz der Wörter. Bei dieser letzten Feststellung ist zu beachten, daß quantitative Änderungen noch keine Antwort geben auf die Frage nach den qualitativen Änderungen. Hier muß also noch einiges ergänzt werden.

Schlosser hat das Problem der sprachlichen Unterschiede zwischen Ost und West in seinem Buch systematisch behandelt und ist dabei auf einige wichtige Sparten der Gesellschaft eingegangen: die "sozialistische Volkswirtschaft", die "sozialistische Erziehung", den Journalismus in der DDR, die Sprache der "Kulturschaffenden". Zur Veranschaulichung des Gedankens, daß fehlende Kenntnisse über die Sprache des östlichen, beziehungsweise westlichen Gesprächspartners zu Kommunikationsproblemen führen können und in direkter Beziehung stehen zu weltanschaulichen, ideologischen Differenzen, greife ich drei Phănomene heraus.

\subsection{Neue Worrter ideologisch bedingt}

Die Einführung neuer Wörter in der Sowjetischen Besatzungszone und später in der DDR war selbstverständlich ideologisch bedingt. Für diese sprachlichen Innovationen gibt Schlosser drei Herkunftsbereiche an:

Unmittelbare Übernahme von Russismen in Form von Fremdwörtern und Lehnbildungen, Einführung von Begriffen und Formeln, die deutsche und sowjetische Kommunisten aus gemeinsamem Gebrauch kannten und die teilweise auf sprachliche Traditionen der internationalen Arbeiterbewegung zurückgingen, (Wieder-) Verwendung des Sonderwortschatzes der politischen Sprache, die die deutschen Kommunisten bis 1933 und auch 
noch spăter in Widerstand und Untergrund benutzt hatten (Schlosser, 1990:23).

Wörter, die übernommen wurden, waren etwa: Havarie, Brigade, Kombine, Magistrale, Subbotnik.

\subsection{Die Verwendung von Abkürzungen in der DDR}

Eine andere Erscheinung, die vermutlich viel stärker ins Leben des Alltags eingriff und wie jahrelange Erfahrungen mich lehrten, die Kommunikation zwischen Ost und West sehr auf die Probe stellen konnte, war die Verwendung von Abkürzungen in der DDR. Aus Gründen der Sprachenergie waren diese Abkürzungen unerläßlich. Es gab soviele gesellschaftliche Einrichtungen, deren Namen ein ideologisches Programm beinhalteten, da $\beta$ eine Abkürzung dieser Bezeichnungen auf der Hand lag. Ich nenne nur einige wenige aus der Fülle der sogenannten Akü-Wörter: VEB (Volkseigener Betrieb), HO (Handels-Organisation), LPG (Landwirtschaftliche Produktionsgenossenschaft), FDJ (Freie Deutsche Jugend), ABF (Arbeiter-und Bauern-Fakultat), FDGB (Freier Deutscher Gewerkschaftsbund). Das Kulturpolitische Worterbuch der DDR ist eine Fundgrube für Beispiele solcher Abkürzungen (Berger, 1978). Jeder Mensch wird mal die Erfahrung gemacht haben, daß von anderen verwendete $A b-$ kürzungen, die man nicht auf ihre vollständige Bedeutung zurückführen konnte, als Blockade, als Mittel der Exklusivităt wirkten. So haben diese DDR-Abkürzungen in der Praxis der sprachlichen Kommunikation funktioniert. Für Westdeutsche war die FDJ fremd. Und wenn die Abkürzung vertraut war, graute man sich vor diesem Phänomen, da man sie direkt mit einer anderen Abkürzung, der HJ nämlich, assoziierte. Leider bagatellisiert Drosdowski die Folgen solcher sprachlichen Charakteristika zu sehr in seinem von mir erwähnten Vortrag. Der negative Effekt auf die Kommunikation war um so größer, da man ja im Prinzip davon ausging, sich als West- und Ostdeutsche sprachlich verständigen zu können. In der Praxis fuihrte dies in den meisten Fällen zur Kapitulation der Ostdeutschen, die ja nicht gerne ihre Rolle als Empfänger auf materiellem Gebiet einbüßen wollten. Die Folge ist ein Gefühl sprachlicher Entfremdung.

\subsection{Die marxistische Tendenz zur religiősen Entfremdung}

Die dritte Erscheinung bezieht sich auf die erklärte Tendenz des marxistisch regierten Staates, die Religion durch gesellschaftliche Änderungen überflüssig zu machen und religiöse Institutionen und Feiern durch sozialistische Einrichtungen und Festakte zu ersetzen. So war die Regierung der DDR bestrebt, an die Stelle der evangelischen Konfirmation und der katholischen Firmung die Jugendweihe zu stellen. Das Kulturpolitische Worterbuch gibt folgende Umschreibung der Jugendweihe: 
... gesellschaftliche Einrichtung in der DDR zur Unterstützung der kommunistischen Erziehung der Jugendlichen im 8. Schuljahr die Jugendweihe knüpft an eine Tradition der revolutionären deutschen Arbeiterbewegung an und entwickelt sie weiter. Als Teil des einheitlichen sozialistischen Bildungswesens trägt die Jugendweihe dazu bei, den Jugendlichen anwendungsbereites Wissen in Grundfragen der wissenschaftlichen Weltanschauung und der sozialistischen Moral zu vermitteln, sie im Geiste des sozialistischen Patriotismus und proletarischen Internationalismus zu erziehen und ihnen zu helfen, sich auf die aktive Teilnahme an der Gestaltung der entwickelten sozialistischen Gesellschaft und an der Schaffung grundlegender Voraussetzungen für den allmählichen Übergang zum Kommunismus vorzubereiten (Kulturpolitisches Wörterbuch; vgl. Berger, 1978:321).

Zur Vorbereitung auf die Jugendweihe dienten die sogenannten Jugendstunden. Während der Jugendweihe wurde das Jugendweihe-Gelöbnis abgelegt. Für die Hintergründe des Verhältnisses zwischen Staat und Kirchen in der DDR (und für religiose Entfremdung) verweise ich auf folgende Publikationen: The Lutheran Church and the East German State (Goeckel, 1990); Dies ist nicht unser Haus. Die Rolle der katholischen Kirche in den politischen Entwicklungen der DDR (Ester et al., 1992); Der SED-Staat und die Kirche (Besier, 1993).

Die politische Tendenz, das Kirchlich-Religiöse durch Pseudo-Religiöses zu ersetzen, fand einen deutlichen Ausdruck in den 1958 von Walter Ulbricht initiierten "Zehn Geboten der sozialistischen Moral":

1. Du sollst Dich stets für die internationale Solidarität der Arbeiterklasse und aller Werktätigen sowie für die unverbrüchliche Verbundenheit aller sozialistischen Länder einsetzen.

2. Du sollst Dein Vaterland lieben und stets bereit sein, Deine ganze Kraft und Fähigkeit für die Verteidigung der Arbeiter-und-Bauem-Macht einzusetzen.

3. Du sollst helfen, die Ausbeutung des Menschen durch den Menschen zu beseitigen.

4. Du sollst gute Taten für den Sozialismus vollbringen, denn der Sozialismus führt zu einem besseren Leben für alle Werktätigen.

5. Du sollst beim Aufbau des Sozialismus im Geiste der gegenseitigen Zusammenarbeit handeln, das Kollektiv achten und seine Kritik beherzigen.

6. Du sollst das Volkseigentum schützen und mehren. 
7. Du sollst stets nach Verbesserungen Deiner Leistungen streben, sparsam sein und die sozialistische Arbeitsdisziplin festigen.

8. Du sollst Deine Kinder im Geiste des Friedens und des Sozialismus zu allseitig gebildeten, charakterfesten und körperlich gestählten Menschen erziehen.

9. Du sollst sauber und anständig leben und Deine Familie achten.

10. Du sollst Solidarität mit den um ihre nationale Befreiung kämpfenden und den ihre nationale Unabhängigkeit verteidigenden Völkern üben (DDR-Handbuch, 1975:577f).

Auf diese Beispiele sprachlicher Divergenz zwischen Ost und West möchte ich mich beschränken. Sie wollten zeigen, daß der spezifısche sozialistische Wortschatz der DDR keine sprachliche Verbrämung eines unverwandelten Herzens der deutschen Sprache bildete, sondern Kernbereiche des Denkens, des Fühlens und des Glaubens berührte. Wenn diese Prämisse akzeptiert wird, dann ist die notwendige Schlußfolgerung die, da $\beta$ Kenntnisse dieser so gefärbten Sprache unbedingt notwendig sind, um den Gesprächspartner von jenseits der Elbe in seiner historischen Verwurzelung zu verstehen. Die Dokumente der DDR, einschlie $\beta$ lich der in der DDR entstandenen Literatur, werden in einem Tempo veralten, sie werden so rasch den Charakter der Unzeitgemåßheit annehmen, da $\beta$ der Leser des nächsten Millenniums keinen Zugang mehr $\mathrm{zu}$ ihnen finden wird. Diesen Proze $\beta$ des Veraltens gilt es aufzuhalten, da die Geschichte meines Partners die Voraussetzung für unsere gemeinsame Zukunft ist. Die Frage ist, ob ein derartiges Unternehmen gelingen kann. Das wurde mir stark bewußt bei der Lektüre der Memoiren des wichtigsten Kulturideologen der DDR Kurt Hager: Erinnerungen (1997). An diesem Buch ist der Proze $\beta$ der Sprachveränderung deutlich abzulesen, vergleichbar mit dem Effekt der mit Erich Honecker geführten Gespräche, die 1990 unter dem Titel Der Sturz (1990) veröffentlicht wurden. Das Problem der Entfremdung zwischen Ost und West steht im Mittelpunkt des von Willem Melching und Leo Paul im Jahre 1997 im Deutschland-Institut der Universităt Amsterdam veröffentlichten Buches De integratie van Oost-en WestDuitsland.

\section{Ist DDR-Nostalgie zu rechtfertigen?}

Darf man der DDR nachtrauern? Ich glaube, "nachträuern" ist ein zu großes Wort. Dieses Zeitwort impliziert, da $\beta$ man um etwas Verlorenes trauert und da $\beta$ man sich das Verlorengegangene zurückwünscht. Aber wenn "nachtrauem" keine adăquate, auf Zukunft gerichtete Haltung darstellt, dann ist noch immer die Legitimität der Trauer zu verteidigen. Das Vergangene mu $\beta$ ja verarbeitet werden. In dieser Verarbeitung liegt die Botschaft des Psychoanalytikers Hans- 
Joachim Maaz mit seinem Buch Der Gefühlsstau - ein Psychogramm der DDR aus dem Jahre 1991. Um das seelische Gleichgewicht nicht zu verlieren oder um es wiederzugewinnen, ist Einsicht in die Voraussetzungen der eigenen Geschichte lebensnotwendig. Dazu ist auch vonnöten, da $\beta$ der positive Aspekt meiner Lebensorientierung in einer marxistischen Gesellschaft anerkannt wird. Das kann der Westdeutsche nur, wenn er die Aufgabe übernimmt, sich das Leben in der DDR nachträglich anzueignen. Der beste Weg dazu ist nach wie vor die Literatur, namentlich die Romane der Autorin Christa Wolf von Der geteilte Himmel (1963) bis Sommerstück (1989).

Der Essener Psychotherapeut Jorgos Canacakis hat in seinem Buch über das Trauern substantielle Dinge gesagt, die in der gegenwärtigen geschichtlichen Situation hilfreich sind. Ich zitiere aus seinem Buch Ich sehe Deine Tränen einen Absatz, der mir sehr passend erscheint für das in diesem Beitrag angesprochene Thema:

Trauer hat es schwer in einer Zeit, in der wir durch den technisch raschen Fortschritt gezwungen werden, von alltäglichen Dingen, von vertrauten Gewohnheiten Abschied zu nehmen. Trauer ist nicht 'in'. Unsere idealisierten Schöpfungen, die Maschine und der Computer, weinen und trauern nicht. Diese Gesellen aus Eisen, Glas und Plastik werden repariert, ersetzt oder verschrottet, ohne da $\beta$ es Probleme gibt. Um sie brauchen wir uns keine 'Trauersorgen' zu machen. Sobald wir uns aber mit diesen blo $\beta$ funktionierenden Dingen nicht auf eine Stufe stellen wollen, haben wir Sorge, mit dem Gefühl der Trauer zurechtzukommen (Canacakis, 1987:14).

Die gesellschaftliche Realität innerhalb der ehemaligen Deutschen Demokratischen Republik veranschaulicht die Wahrheit dieses Satzes.

\section{Bibliographie}

Berger, Manfred (Hrsg.). 1978. Kulturpolitisches Wörterbuch. Zweite Auflage Berlin Dietz Verlag.

Besier, Gerhard 1993. Der SED-Staat und die Kirche. München : Bertelsmann Verlag.

Canacakis, Jorgos. 1987. Ich sehe Deine Tränen. Trauern, Klagen, Leben können. Stuttgart Kreuz Verlag.

DDR-Handbuch. 1975. Herausgegeben vom Bundesministerium fur innerdeutsche Beziehungen. Köln : Verlag Wissenschaft und Politik.

Drosdowski, Günther. 1991. Rede. In: Duden. Die Reden anlaßlich der Vorstellung des 'wiedervereinigten' Wörterbuchs zur deutschen Rechtschreibung. Mannheim/Leipzig/ Wien/Zürich. S. 11-16.

Ester, H. et al. 1993. Dies ist nicht unser Haus. Die Rolle der katholischen Kirche in den politischen Entwicklungen der DDR. Amsterdam : Editions Rodopi. (= German Monitor 28.)

Goeckel, Robert F. 1990. The Lutheran church and the East German State. Ithaca/London Cornell University Press.

Hager, Kurt. 1997. Erinnerungen. Leipzig : Faber \& Faber. 
Honecker, Erich. 1990. Der Sturz. Berlin \& Weimar : Aufbau Verlag.

Krabbe, Katrin. 1993. Gespräch mit der Athletin Katrin Krabbe. Zeit-Magazin: 13, März 26.

Labroisse, Gerd, \& Wallace, lan. 1991. DDR-Schrifisteller sprechen in der Zeit. Eine Dokumentation. Amsterdam : Editions Rodopi. (= German Monitor 27.)

Maaz, Hans-Joachim. 1991. Der Gefühlsstau. Ein Psychogramm der DDR. Berlin : Argon Verlag.

Melching, Willem \& Paul, Leo. 1997. De integratie van Oost- en West-Duitsland. Amsterdam : Duitsland Instituut, Universiteit van Amsterdam.

Michaelis, Rolf. 1991. Wir bauen die neue Mauer. Ein Bericht aus Weimar. Die Zeit: 22, Mai 24.

Reich, Jens Georg. 1992. Allein es fehlt die Botschaft. Zeit-Magazin: 5, Januar 24.

SBZ. 1949.

Vergleich

Die SBZ von $A$ bis $Z$. Ein Taschen- und Nachschlagebuch über den anderen Teil Deutschlands. Herausgegeben vom Bundesministerium für gesamtdeutsche Fragen. Bonn : Bundes-Verlag.

Schlosser, Horst Dieter. 1990. Die deutsche Sprache in der DDR zwischen Sialinismus und Demokratie. Historische, politische und kommumikative Bedingungen. Köln : Verlag Wissenschaft und Politik.

Schmidt, Helmut. 1993. Wir sind noch kein normales Volk. Die Zeit: 14, April 2.

Schorlemmer, Friedrich. 1992. Worte öfnen Fäuste. Die Rückkehr in ein schwieriges Vaterland. München : Kindler Verlag.

Schorlemmer, Friedrich. 1993. Den Frieden riskieren. In: Friedenspreis des Deutschen Buchhandels 1993. Friedrich Schorlemmer. Frankfurt am Main : Börsenverein des Deutschen Buchhandels.

Voigt, Jutta. 1991. Blitzkrieg gegen die Erinnerung. Zeit-Magazin: Okt. 25.

Wolf, Christa. 1989. Sommerstück. Berlin \& Weimar : Aufbau Verlag.

Wolf, Christa. 1963. Der geteilte Himmel. Halle/Saale : Mitteldeutscher Verlag. 
